\title{
Influence of Voltage on Electrolysis and Plasma Polishing Ji Wang ${ }^{1,{ }^{*}}$, Xue-mei Zong ${ }^{1}$, Jian-fei Liu ${ }^{1,{ }^{*}}$ and Sen Feng ${ }^{1}$ \\ ${ }^{1}$ Jiangsu Xuzhou Construction Machinery Research Institute, Xuzhou, China
}

Keywords: Electrolysis and Plasma, Voltage, Material Removal Rate, Roughness, Electrical Power.

\begin{abstract}
Influence of voltage on material removal rate, surface roughness and power consumption were experimentally studied in this paper. Different polishing states were analyzed from standpoint of electricity transition on the basis of the polishing mechanism. The reason that material removal rate, surface roughness and electricity cost influenced by voltage was given. The experiments show that, material removal rate gradually decrease and velocity of surface roughness reduction gradually slow with increase of voltage. When voltage is at a range of $270-330 \mathrm{~V}$, the minimum of surface roughness that polishing can reach is the minimum; when voltage is $270-290 \mathrm{~V}$, electrical power reaches the minimum.
\end{abstract}

\section{Introduction}

Metal surface electrolysis and plasma polishing(EPP) can polish metal workpieces by generating plasma gas encapsulating the workpieces based on the vapor-liquid techniques of plasma generation. Compared with traditional polishing method, the technique with the characteristics such as polishing no mechanical stresses, high machining efficiency and high uniform polishing quality is more applicable for the workpieces with complex shape. It has been used in machinery manufacturing, aviation industry and automobile manufacturing, with a far-ranging market prospect[1,2,3].

Polishing solution of EPP is salt solution with low concentration (3-6wt\%), which is non-pollution and cheap,therefore cost of EPP mainly depends on the power consumption. The problem of how to reduce the consumption and improve the polishing efficiency is always an important research task in EPP [4,5]. Voltage as a key parameter is controlled within a range of 200-400V[6]. But there have been no reports of influence of Voltage on EPP.

In this paper, influence of voltage on surface roughness, material removal rate and power consumption were experimentally studied, then the variation of them with voltage was obtained and the effects of the voltage changes in EPP was analyzed based on mechanism of EPP.

\section{Polishing Mechanism}

In EPP, workpieces are connected to the positive pole of the power supply, polishing solution and bath are the negative pole. The workpiece is soaked into polishing solution making the polishing solution electrolyzed first and producing a small amount of gas. The workpiece contacts to the polishing solution resulting in instantaneous short circuit of the system. Electrons and ions are harder to move near solid-liquid interface, where resistance value is largest and a large amount of heat quantity is released. Some of this heat makes the polishing solution evaporated to form a gas layer segregating the workpiece and the polishing solution completely and ending the short circuit of the system, and the rest drives up the temperature of the workpiece. The breakdown and discharge in the gas layer under DC Voltage make neutral particles be ionized. The electrons moving toward the anode at high speed collide with neutral particles to produce new electrons and positive ions, so the amount of electrons increases like avalanche (electron avalanche), forming the whole discharge channel with high-density charged particles. The collision between the workpiece surface and electrons moving at high speed in the discharge channel produces a large amount of heat quantity which causes surface material of the workpiece to melt and removed $[7,8]$. 


\section{Experimental Process}

The material during the polishing was SUS304 stainless steel and was cut into four test specimens of $40 \times 50 \times 4 \mathrm{~mm}^{3}$. Polishing was performed by using a self-made $100 \mathrm{kw}$ EPP machine, whose polishing bath has a volume of $400 \mathrm{~L}$. Ammonium sulfate solution with concentration $3 \mathrm{wt} \%$ was chose as polishing solution. The test specimens were rubbed with 400 mesh sandpaper for 20 minutes and cleaned with deionized water before the experiment; make sure that the surface roughness, as shown in Table.1, is approximately equal.

Table 1. Surface roughness before experiment

\begin{tabular}{|c|c|c|c|c|}
\hline & Sample1 & Sample2 & Sample 3 & Sample4 \\
\hline Position $1[\mu \mathrm{m}]$ & 0.41 & 0.37 & 0.37 & 0.39 \\
\hline Position $2[\mu \mathrm{m}]$ & 0.38 & 0.43 & 0.37 & 0.41 \\
\hline Position $3[\mu \mathrm{m}]$ & 0.39 & 0.39 & 0.40 & 0.38 \\
\hline Average $[\mu \mathrm{m}]$ & 0.39 & 0.40 & 0.38 & 0.39 \\
\hline
\end{tabular}

Four test specimens were polished at different voltage range, which is $220-250 \mathrm{~V}, 270-290 \mathrm{~V}$, $300-330 \mathrm{~V}$ and $360-390 \mathrm{~V}$ respectively, while the solution temperature was constant at $17^{\circ} \mathrm{C}$. Polishing lasted about 50 minutes, during which time the current value was recorded and the test specimen was taken out to measure the thickness and surface roughness every one minute. Material removal rate was calculated by the thickness variation and polishing time.

\section{Experiment Result and Analysis}

Varying Law of Material Removal Rate with Voltage.Varying curves of the material removal rate with the voltage are shown in Fig. 1. The voltage being kept constant, the material removal amount with the polishing time increases linearly, showing that EPP can remove material very stablely. When the voltages are different, the material removal rate decreases with the increase of the voltages, and reaches a maximum in the $220-250 \mathrm{~V}$ voltage range.

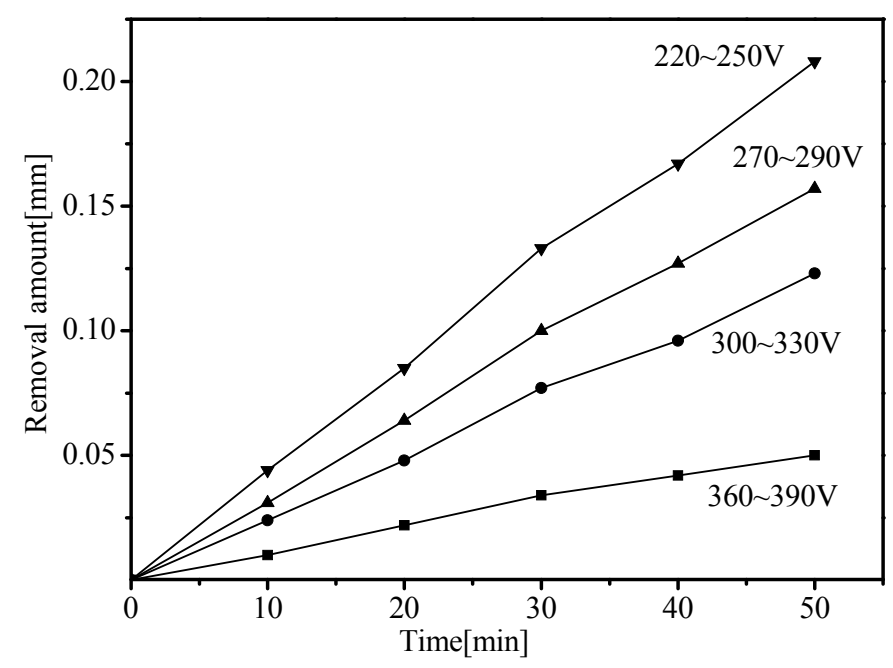

Figure 1. Curve of removal amount versus voltage

The test specimen is supposed to be long thin cylinder, whose radius is $r$ and height is $h$, ignoring the top and bottom surfaces. According to heat balance equation, there is

$$
\rho c d T=\frac{j^{2}}{K} d t
$$


where $\rho$ is the density of the polishing solution, $\mathrm{c}$ is the specific heat capacity, $\mathrm{T}$ is the temperature of the polishing solution near the gas-liquid interface, $\mathrm{j}$ is the electric current density, $\mathrm{K}$ is the electrical conductivity. From

$$
\mathrm{j}=\frac{\mathrm{I}}{\mathrm{S}}=\frac{\mathrm{I}}{2 \pi \mathrm{rh}}
$$

we get

$$
\rho c d T=\frac{I^{2}}{4 K \pi^{2} r^{2} h^{2}} d t
$$

equivalent resistance of the gas layer can be expressed as

$$
\mathrm{R}=\frac{\ln \frac{\mathrm{r}_{1}}{\mathrm{r}}}{2 \pi \mathrm{hK}}=\frac{\mathrm{U}_{1}}{\mathrm{I}}
$$

where $r_{1}$ is the radius of outer surface of the gas layer, $U_{1}$ is the voltage across the gas layer, the system voltage is approximately the voltage across the gas layer, while the equivalent resistance of the gas layer is considerably greater than any resistance in the system. Eq. 3 becomes

$$
\rho c d T=\frac{\mathrm{U}^{2} \mathrm{~K}}{\mathrm{r}^{2} \operatorname{In}^{2} \frac{1}{\mathrm{r}}} \mathrm{dt}
$$

Integrating both sides of Eq. 3, we get

$$
\mathrm{t}_{1}=\frac{\rho c\left(\mathrm{~T}_{1}-\mathrm{T}_{\mathrm{o}}\right) \mathrm{r}^{2} \operatorname{In}^{2 \frac{\mathrm{r}_{1}}{\mathrm{r}}}}{\mathrm{U}^{2} \mathrm{~K}}
$$

where $T_{1}$ is vaporization temperature of the polishing solution, $t_{1}$ is the time needed for making the polishing solution temperature rise to vaporization temperature.

The material removal rate is dependent on energy gains of the surface of the specimen per unit time besides its physical properties, such as hardness, melting point, electrical conductivity. Energy gains per unit time become an important determinant of the material removal rate because of the four test specimens with the same materials.

In the moment the specimen contacts with the polishing solution, because of instantaneous short circuit of the system, a large amount of heat quantity is released and the polishing solution is evaporated to form a encapsulating the workpiece. The gas moves up along the surface of the specimen until it is separated from the specimen on one hand, and the instantaneous short and the collision of the electrons with the neutral particles produce heat and vaporize the polishing solution to create gas on the other hand. The gas layer tends to be a relatively steady state during the polishing process. So thickness of the gas layer depends energy gain on the surface of the specimen per unit time, and the more the energy gain is, the thicker the gas layer is.

It can be shown from Eq. 6 that the time needed for making the polishing solution temperature rise to vaporization temperature is shorter when the voltage is higher, so creating more gas leads to thicker gas layer. Conversely, forming thinner gas layer causes increasing probability of contact between the specimen and the polishing solution, because the gas layer is relatively stable and changes at any moment. Once any location on the specimen contact with the polishing solution, a large amount of heat generated within a short time removes the material rapidly at this location.

Above all, at high voltages, the gas layer is thick and stable, where spark discharge will be generated, so the material removal rate is low. With the decrease of the voltage, the gas layer tends to thinner and probability of the short circuit increases, so energy the gain per unit time and the material removal rate increase. Of course, if the voltage is too low to maintain EPP, the specimen will be electrolyzed in the solution and the material removal rate becomes low. 
Varying law of surface roughness with voltage.In the first five minutes, the roughness value decreases quickly with the voltage being constant and then roughness gradient became smaller, as shown in Fig. 2 and Fig. 3. The roughness value descends most quickly from $\mathrm{Ra}=0.4 \mu \mathrm{m}$ to $\mathrm{Ra}=0.17 \mu \mathrm{m}$ in the $220-250 \mathrm{~V}$ voltage range, and most slowly in the $360-390 \mathrm{~V}$ voltage range.

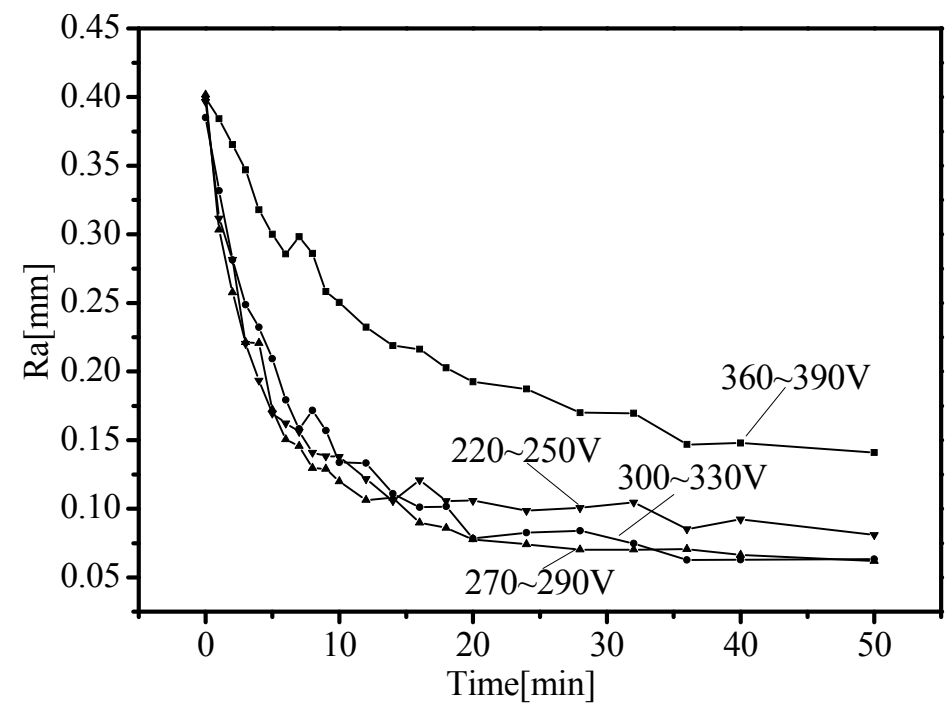

Figure 2. Curve of surface roughness with time (50 minutes)

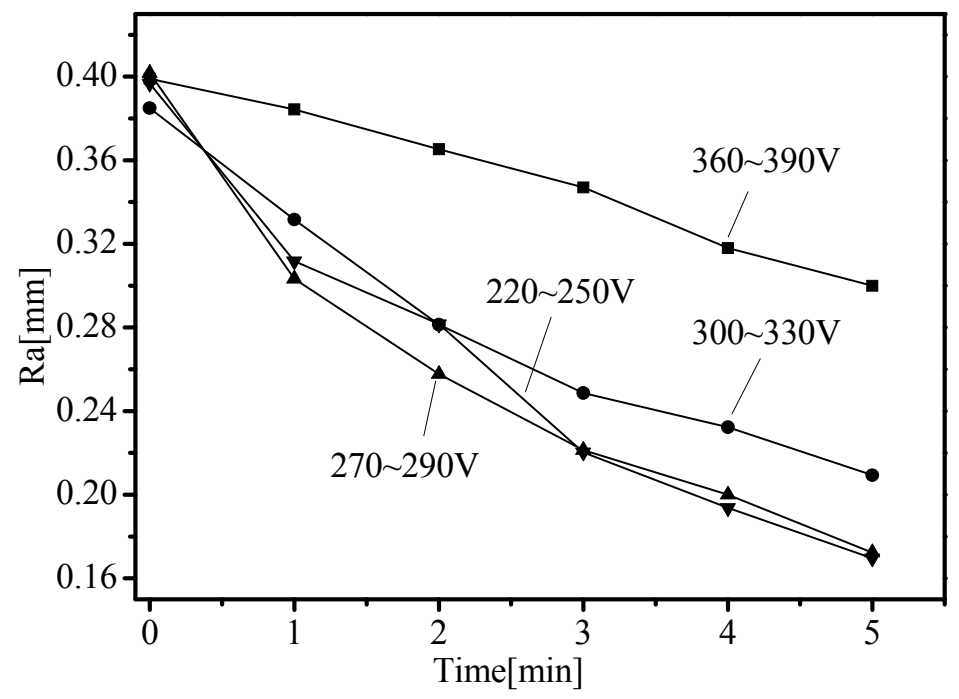

Figure 3. Curve of surface roughness with time (first 5 minutes)

Between the 20th and 50th minute, the roughness value continues the downward tendency obviously, which illustrates that it doesn't to reach the minimum under the process condition in the $220-250 \mathrm{~V}$ voltage range after 50 minutes. In the other voltage range, roughness gradient has been small after 20 minutes, which illustrates that it has approached or reached the minimum. The roughness value in the $270-330 \mathrm{~V}$ voltage range after 50 minutes is the smallest within a range of $0.06-0.08 \mu \mathrm{m}$.

The key to EPP is that the discharge channel preferentially forms in the convex zone of the specimen surface so that the material in the convex zone is removed more. The smallest roughness value that EPP can reach under the process condition depends on the depth of the electric erosion pit formed by one discharge, and the smaller the depth is, the smaller the value is. In the first five minutes, the specimen has a wavy surface obviously, and the electric field intensity in the convex zone is remarkably stronger than in the concave zone. In the beginning stages, the discharge channel mostly forms in the convex zone and the convex on the surface is removed to reduce the surface roughness 
fast. As polishing continue, surface flat gradually takes effect so that the discharge channel forms more randomly and the roughness value descends slowly.

When the voltages are different, the decline rate of the roughness value is also relevant to thickness of the gas layer. Convex and concave on the surface causes more apparent difference of the electric field intensity in condition of the low voltage and thin gas layer. Besides, one discharge will form a shallow electric erosion pit because the electrons gain low energy by electric fields in low voltage. The overall result is that the roughness value descends quickly and the smaller value is obtained. But if the voltage is too smaller, probability of the short circuit will increase leading to a slowdown to the roughness value descending and an increase to the smallest roughness value that EPP can reach under the process condition because energy gains of the surface by the short circuit is more than by the discharge and the short circuit can form a deeper electric erosion pit. It can be seen from Fig. 2 that the rate of the roughness value descending in the $220-250 \mathrm{~V}$ voltage range no faster than in the $270-290 \mathrm{~V}$ voltage range, and there is the smaller limiting roughness value that EPP can reach in the $270-290 \mathrm{~V}$ voltage range.

Effect of voltage on the polishing current and electric power. During the course of the experiment, the current value was recorded to take the average every a minute in order to eliminate the adverse effect of the polishing current fluctuation. From the result of this experiment, the current value doesn't increase with the voltage increases, but decrease evidently and then increase slightly. These results show that the equivalent resistance of the system, which mainly depends on the gas layer, changes with the voltage. The thicker the gas layer is, the bigger the resistance is in general. Fig. 4 and Fig. 5 are the changing curves of the current and electric power with the voltage separately.

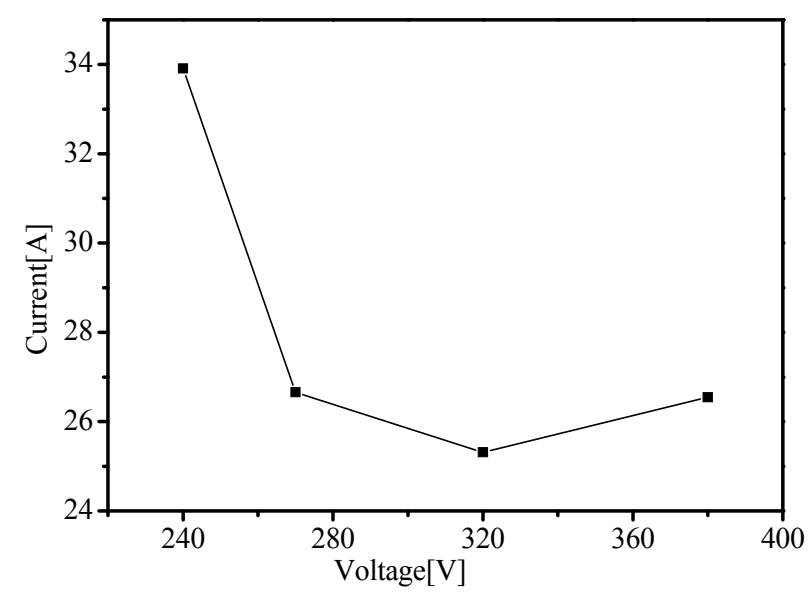

Figure 4.Curve of current versus voltage

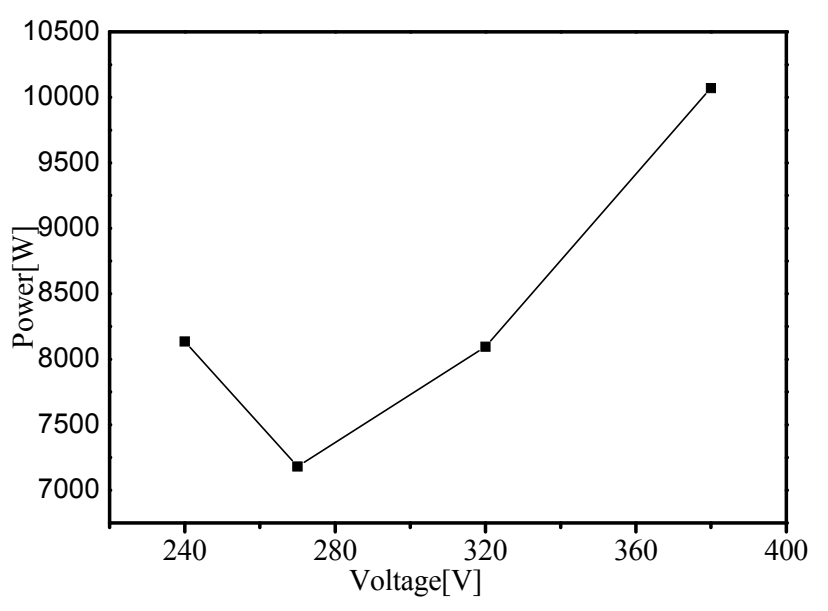

Figure 5. Curve of power versus voltage

In the $220-250 \mathrm{~V}$ voltage range, the current value reaches its maximum of $34 \mathrm{~A}$ with the largest fluctuation. Under the voltage, the gas layer is thin to increase the probability of contact between the polishing solution and the specimen, so the current value and the fluctuation are often large. With the increase of the voltage, the current decreases as the gas layer thickens and the ratio of the short circuit decreases. But, if the voltage increases to some extent, the short circuit will rarely occur. In that case, the current will slightly increase because of the increase of the voltage.

The curve of the electric power has tendency of ascending first and descending in succession, as shown in Fig. 5. The short circuit that occurs frequently in low voltage increases the polishing current and electric power. The gas layer tends towards stability and the electric power can increase to certain value when the voltage increases. After this, influence of the short circuit on the electric power is smaller and smaller. Meanwhile the steadily increasing voltage value plays a more significant role in the electric power so that the electric power increases with increase of the voltage. From the research results it can be seen that the high voltage is detrimental to the polished effect and energy consumption on the premise of forming the stable gas layer. 


\section{Conclusions}

The material removal rate decreases with the increase of the voltages. The short circuit that occurs frequently increases the material removal rate at a low voltage. Increasing the voltage, the gas layer seems to be stable and the material removal rate decrease because the material is mainly removed by spark discharge; The rate of decrease the roughness value slows with the increase of the voltages. When the voltage is at a range of $270-330 \mathrm{~V}$, the smallest roughness value that EPP can reach is the minimum under different voltages. If the voltage less than $270 \mathrm{~V}$, the smallest roughness value increases instead because of the short circuit; The polishing current is the minimum in the $250-330 \mathrm{~V}$ voltage range and the electric power is the minimum in the $270-290 \mathrm{~V}$ voltage range.

\section{References}

[1] H.Y. Weng, Z.H. Li, Several techniques comparision of polishing, Mechanical Engineer. 6 (2010) 28-29.

[2] X.K. Zhao, Z. Wang, Y.F. Zheng, Development on polishing, SurfaceTechnology. 293 (2000) 6-7.

[3] D. Zhu, The latest advance and the principal issues in ECM, Electromachining\&Mould. 1 (2000) 11-16.

[4] J. Wang, L.C. Suo, Effect of Plasma Polishing on the suaface state of stainless steel, Hot Working Technology. 367 (2009) 62-64.

[5] J. Wang, L.C. Suo, Regularity of surface roughness withpolishingtimeinelectrolysisandplasma polishing, J. Harbin Eng. Univ. 342 (2013) 227-232.

[6] J. Wang, L.C. Suo, Y.L. Fu, Detecting the mass fraction of ammonium sulfate in polishing solution in electrolysis and plasma polishing, Materials Science \& Technology. 222 (2014) 30-33.

[7] J. Wang, L.C. Suo, L.L. Guan, Analytical Study on Mechanism of Electrolysis and Plasma Polishing, Advanced Materials Research. 472-475 (2012) 350-353.

[8] J. Wang, L.C. Suo, L.L. Guan, Optimization of Processing Parameters for Electrolysis and Plasma Polishing, Applied Mechanics and Materials. 217-219 (2012) 1368-1371. 